\title{
HADAMARD MATRICES OF ORDER CUBE PLUS ONE
}

\author{
K. GOLDBERG
}

1. Result. Let $A$ be an Hadamard design of type 1, and let $(X, Y, Z)$ denote the direct product of matrices $X, Y$ and $Z$ (the direction of the product is unimportant here). Later we shall show that

$$
\begin{aligned}
B= & (I, A, J)+(J, I, A)+(A, J, I) \\
& +(A, A, A)+\left(A, A^{T}, A^{T}\right)+\left(A^{T}, A, A^{T}\right)+\left(A^{T}, A^{T}, A\right)
\end{aligned}
$$

is also an Hadamard design of type 1 . This construction will prove the theorem:

TheOREM. If there is an Hadamard matrix of type 1 and order $h$, then there is an Hadamard matrix of type 1 and order $(h-1)^{3}+1$.

Williamson [2] shows that there exist Hadamard matrices of type 1 for all orders

$$
\left.2^{a}\left(p_{1}^{a_{1}}+1\right) \cdots\left(p_{r}^{a_{r}}+1\right)\right\} \begin{aligned}
& a, r=0,1,2, \cdots, \\
& a_{1}, \cdots, a_{r}=1,3,5, \cdots,
\end{aligned}
$$

where each $p_{i}$ is a prime congruent to 3 modulo 4 .

For example, an Hadamard matrix of type 1 and order 16 exists. By our theorem, one also exists of order $15^{3}+1=16 \cdot 211$, which is not one of the numbers (1). However, another construction of Williamson [2] yields an Hadamard matrix (not of type 1) for this order. The first "new" order is $39^{3}+1$.

2. Definitions and proof. Throughout this paper $I$ and $J$ denote the identity matrix and the matrix with 1 in every position respectively, of the order required by the context. An $a, b$ matrix is one in which each element is either $a$ or $b$.

An Hadamard matrix is a $1,-1$ matrix $H$ of order $h$ such that $H H^{T}=h I$. (Necessarily either $h=2$ or $h$ is divisible by 4 .) It is of type 1 if $H+H^{T}=2 I$.

An Hadamard design $A$ is a 0,1 matrix of order $h-1$ such that $A A^{T}=A^{T} A=(h / 4) I+(h / 4-1) J$. (Necessarily $A J=J A=(h / 2-1) J$.) It is of type 1 if $A+A^{T}=J-I$.

If $H$ is an Hadamard matrix it can be multiplied by generalized permutation matrices to bring it into the form

Presented to the Society, April 14, 1960; received by the editors February 12, 1964. 


$$
\left(\begin{array}{rr}
1 & 1 \ldots 1 \\
-1 & \\
\vdots & J-2 A \\
-1 &
\end{array}\right)
$$

where $A$ is an Hadamard design. Then $H$ is of type 1 if and only if $A$ is of type 1 .

To prove that $B$ is a 0,1 matrix we write it in the form

$$
B=(I, X)+(A, Y)+\left(A^{T}, Z\right)
$$

where $(P, Q)$ denotes the direct product of $P$ and $Q$, and

$$
\begin{aligned}
& X=(I, A)+(A, J), \\
& Y=(I, I+A)+(A, I+A)+\left(A^{T}, I+A^{T}\right), \\
& Z=(I, A)+\left(A, A^{T}\right)+\left(A^{T}, A\right) .
\end{aligned}
$$

Since $I, A$ and $A^{T}$ are mutually disjoint we need only show that $X, Y$ and $Z$ are 0,1 matrices. And the same reasoning, applied to each, confirms this.

To prove that $B+B^{T}=J-I$ we need only note that

$$
X+X^{T}=J-I \text { and } Y+Z^{T}=Y^{T}+Z=J .
$$

It remains to prove that $B B^{T}$ is a linear combination of $I$ and $J$. This is straightforward algebraic manipulation. First

$$
B B^{T}=(I, U)+(A, V+(n-1) W)+\left(A^{T}, V^{T}+(n-1) W\right)
$$

where

$$
\begin{aligned}
U & =X X^{T}+(2 n-1)\left(Y Y^{T}+Z Z^{T}\right), \\
V & =X Z^{T}+Y X^{T}+Z Y^{T}, \\
W & =(Y+Z)(Y+Z)^{T}
\end{aligned}
$$

and $n=h / 4$. Evaluating $U, V$ and $W$ we obtain

$$
\begin{aligned}
U & =m I+(m-1) J, \\
V & =-(n-1)(4 n-1) I+6 n(2 n-1) J+(n-1)(I, J), \\
W & =(4 n-1) I+(4 n-1)^{2} J-(I, J),
\end{aligned}
$$

where $m=\left((h-1)^{3}+1\right) / 4$. It follows that

$$
B B^{T}=m I+(m-1) J .
$$

This completes the proof of the theorem. 
It is clear that three different Hadamard designs of type 1 of the same order can be used in constructing $B$. However, all attempts to apply this method using designs of different orders, have failed.

\section{REFERENCES}

1. A. T. Butson, Generalized Hadamard matrices, Proc. Amer. Math. Soc. 13 (1962), 894-898.

2. J. Williamson, Hadamard's determinant theorem and the sum of four squares, Duke Math. J. 11 (1944), 65-81.

National Bureau of Standards 